\title{
Vigencia de la «banda típica» de la provincia de Chumbivilcas, departamento de Cuzco, Perú
}

\section{Hubert Ramiro Cárdenas Coavoy}

Investigador independiente

hubertacrus@gmail.com

\section{RESUMEN}

El presente texto es una aproximación etnográfica y sonora a la «banda típica», una de las agrupaciones musicales tradicionales con mayor presencia en las festividades de los distritos y comunidades campesinas de la provincia de Chumbivilcas, departamento de Cuzco, Perú. Se trata de un trabajo descriptivo que examina los lugares donde participa la banda, la manera de vestir de sus integrantes, su conformación instrumental y organización, la materia de los temas musicales que interpretan y sus toques característicos, ejemplificados con archivos sonoros registrados durante el trabajo de campo y almacenados en internet para acompañar este artículo.

El trabajo de campo se inició en marzo de 2015 y recaba información obtenida mediante observación participante, conversaciones informales, asi como entrevistas individuales y grupales efectuadas a músicos tradicionales y populares en el marco del aniversario de la provincia de Chumbivilcas (21 de junio, distrito de Santo Tomás); de la fiesta patronal de la Virgen de la Natividad (8 de setiembre, distrito de Santo Tomás) y de la fiesta patronal del Señor Justo Juez de Patapampa (día de «compadres», comunidad campesina de Pfuisa, distrito de Llusco). Entre los principales hallazgos se observa que la banda típica ha logrado adaptarse a los vertiginosos cambios del tiempo debido a la estrecha ligazón que guarda con el turupukllay

* El presente texto es un avance del trabajo de investigación con el que optaré al título en la Maestría en Musicología de la Pontificia Universidad Católica del Perú (PUCP). 
o juego de los toros, celebrado en las principales festividades de la provincia de Chumbivilcas.

Palabras clave: música tradicional, banda típica, turupukllay, wakawaqra, Chumbivilcas, Cuzco.

\section{An overview of the «banda típica» (traditional band) of the province of Chumbivilcas, department of Cuzco, Peru}

SUMMARY

This text is an ethnographic and sound approach to the "banda tipica» (traditional band), one of the traditional musical groups with the greatest presence in the festivities of the districts and peasant communities of the province of Chumbivilcas, department of Cuzco, Peru. It is a descriptive work that examines the places where the band participates, the manner of dressing of its members, its instrumental composition and organization, the subject of the musical themes they interpret and their characteristic toques, exemplified by sound files recorded during field work and stored on the Internet to accompany this article.

The field work began in March 2015 and gathers information obtained through participant observation, informal conversations, as well as individual and group interviews with traditional and popular musicians in the framework of the anniversary of the province of Chumbivilcas, (June 21, district de Santo Tomás); festivity of the of the Virgin of the Nativity, (September 8, district of Santo Tomás) and festivity of the of the Señor Justo Juez de Patapampa, (day of "compadres", peasant community of Pfuisa, district of Llusco). Among the main findings is that the typical band has managed to adapt to the vertiginous changes of time due to the close link that it keeps with the turupukllay or "game of bulls», custom that is celebrated in the main festivities of the province of Chumbivilcas.

Keywords: Traditional music, traditional band, turupukllay, wakawaqra, Chumbivilcas, Cuzco. 


\section{INTRODUCCIÓN}

La «banda típica» es una de las agrupaciones musicales de mayor arraigo y vigencia en los distritos y comunidades campesinas de la provincia de Chumbivilcas, departamento de Cuzco, Perú.

Su participación está ceñida tradicionalmente a la animación de fiestas patronales y su evento principal, el turupukllay o juego de los toros, corrida costumbrista en la que no se hiere, tortura, ni asesina al animal.

Conformada instrumentalmente por una de las tantas adaptaciones locales de la foránea banda de origen militar que arribó al Perú antes de su independencia, está integrada por cornetas, pitos (flauta travesera de caña o tubo de plástico), tambores, bombo y wakawaqras (cornetas de cacho).

Realizado desde marzo del año 2015 y aún en desarrollo, el presente texto es una aproximación etnográfica y sonora a la banda típica de la provincia de Chumbivilcas.

\section{ASPECTOS GEOGRÁFICOS Y SOCIALES}

La provincia de Chumbivilcas está ubicada en el departamento de Cuzco, donde colinda con las provincias de Acomayo, Canas, Espinar y Paruro. Además, limita con la provincia de Cotabambas, departamento de Apurímac, y con las provincias de Caylloma y Condesuyos, departamento de Arequipa. Actualmente está integrada por ocho distritos: Capacmarca, Chamaca, Coquemarca, Livitaca, Llusco, Quiñota, Santo Tomás y Velille.

Ubicada en una zona geográfica de difícil acceso, durante los siglos XIX y XX la provincia de Chumbivilcas permaneció, en gran medida, aislada de los cambios modernizadores que comenzaron a transformar al Perú después de su independencia, sobre todo desde las últimas décadas del siglo XIX, cuando 
se evidenció la paulatina institucionalización del Estado y el incremento de su capacidad operativa a partir de empréstitos; el ingreso de capitales extranjeros, bancos y sociedades anónimas que promovieron actividades agrícolas, mineras, comerciales; el desarrollo de ideas progresistas en pro de los derechos laborales y defensa de la tierras de los pueblos originarios; la flexibilización en la movilización territorial de grupos humanos, y la diversificación de la estructura social, entre otros cambios que sentaron las bases del Perú contemporáneo (Matos, 1984, pp. 23-26).

Durante gran parte del siglo XX, la provincia mantuvo dicha condición de aislamiento, aunada al sistema de hacienda, que continuó con las prácticas de producción pecuaria, agraria y minera preindustriales utilizadas desde el período colonial; de acceso a bienes y servicios mediante la economía de mercado, fundamentada en el uso de la moneda, y la economía de subsistencia, basada en el consumo propio, la reciprocidad y el intercambio, así como de culto religioso sincrético y cosmovisión (Villena, 1987).

Mapa 1. Departamento de Cuzco

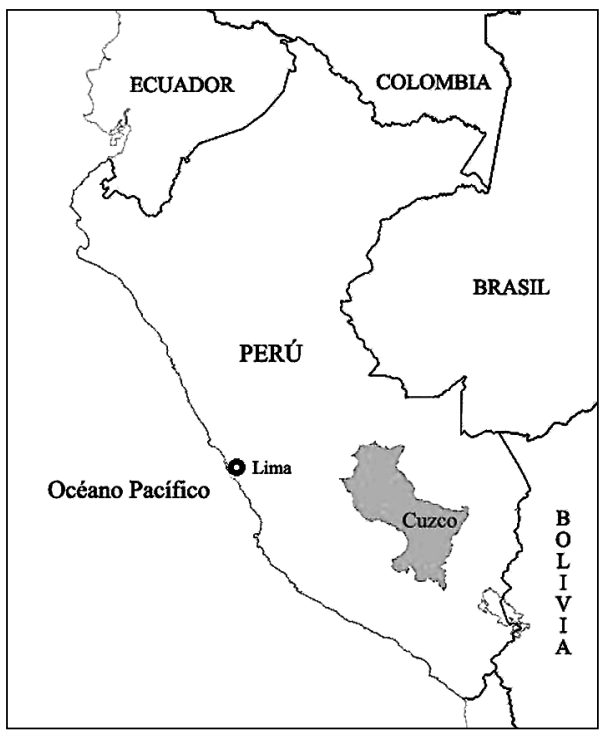

Mapa 2. Provincia de Chumbivilcas, departamento de Cuzco

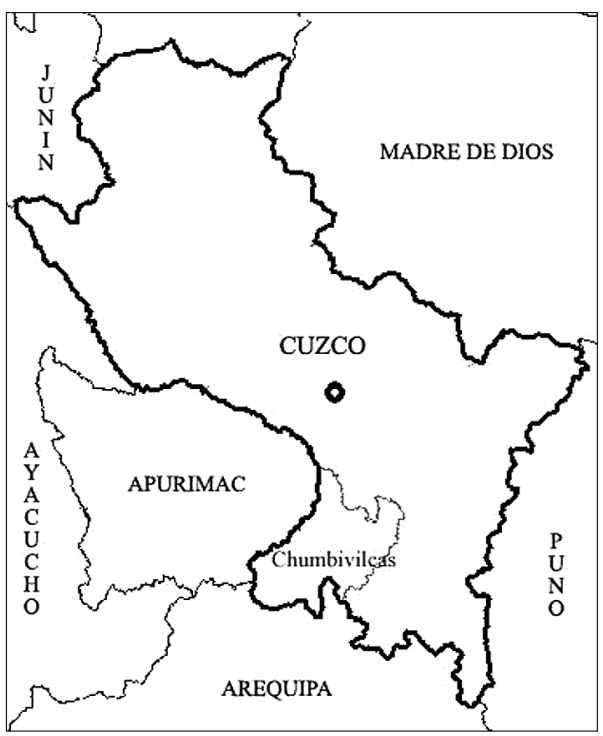


A partir de la segunda mitad del siglo XX, se inicia y concreta un proceso que renovaría por completo la anquilosada estructura socioeconómica de la provincia de Chumbivilcas y gran parte del Perú rural. La rebelión campesina, iniciada el año 1962 en la provincia de La Convención, departamento de Cuzco, como resultado del atraso, abuso y explotación de la población originaria por el sistema de hacienda, la influencia de la revolución cubana sobre los movimientos sociales de izquierda y el desgaste del poder del sector terrateniente (Tamayo, 1978, pp. 232), asociadas a las reformas concernientes a la tenencia de la tierra impulsadas por el Estado, desencadenaron en el año 1969, durante el gobierno del general Juan Velasco Alvarado, la Ley de Reforma Agraria.

Pese a sus deficiencias, esta medida implementada en la provincia de Chumbivilcas desde el año 1974 logró trastornar la prolongada relación dominación-subordinación arraigada en el ámbito rural, reivindicando la tierra de las comunidades campesinas, desintegrando en gran medida el poder terrateniente e iniciando un lento proceso de modernización y democratización que posibilitó el ejercicio de libertades sociales, económicas y culturales del sector campesino.

\section{ACTIVIDAD GANADERA}

Situada aproximadamente entre los 2550 y los 5438 metros sobre el nivel del mar (Gobierno Regional Cusco, 2013, p. 13), la extensión territorial de la provincia de Chumbivilcas comprende una variada gama de recursos naturales $y$, por ende, posibilidades agropecuarias y mineras repartidas en los pisos ecológicos comprendidos en este rango de altitud.

A grandes rasgos, el piso ecológico qhiswa, cuya altitud oscila entre los 2550 y los 3500 metros sobre el nivel del mar, se caracteriza por las facilidades que brinda para la producción de maíz, tubérculos, hortalizas, frutales y la crianza de animales menores; el pata o llanura, con altitud promedio de 3800 metros, se caracteriza geográficamente por sus extensas mesetas de pastizales para el pastoreo de ganado vacuno, equino, ovino, caprino y porcino, y la explotación minera. Finalmente, el piso ecológico puna, cuya altura es superior a los 4000 metros de altitud, es propicio para el pastoreo de llamas y alpacas y actividades de explotación minera. De todos ellos, el territorio de mayor extensión en la provincia de Chumbivilcas se encuentra en el piso ecológico pata o llanura, donde se desarrolla la principal actividad social, económica y cultural de la población chumbivilcana: la ganadería (Villena, 1987, pp. 32-39). 


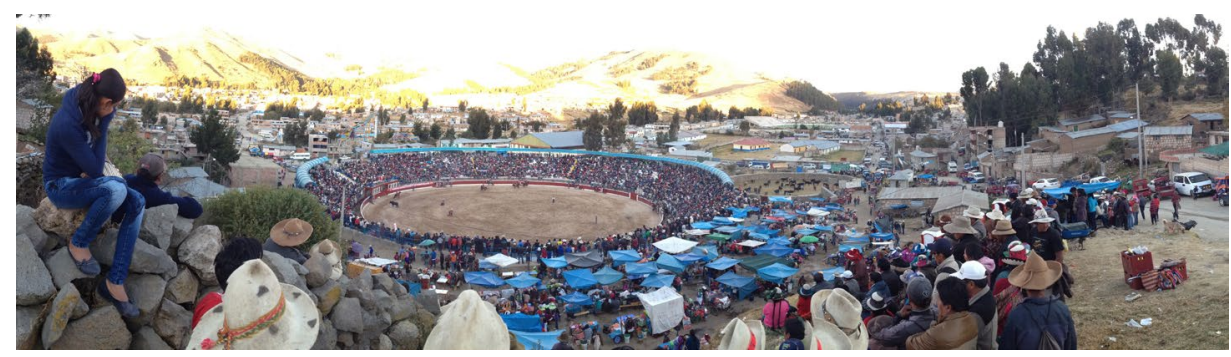

Foto 1. Vista panorámica del turupukllay o juego de los toros, corrida de toros costumbrista donde no se hiere, tortura, ni asesina al animal. Lugar: distrito de Santo Tomás, provincia de Chumbivilcas. Foto: Hubert Ramiro Cárdenas, 2015.

Dicha actividad, cuyos antecedentes se remontan a la época prehispánica, tuvo vital importancia en el Estado inca, donde se disponía de enormes hatos de llamas y alpacas que servían como transporte de carga, materia prima para productos textiles y de cuero, alimento y ofrenda ceremonial. Con la invasión hispana, se introdujeron los ganados vacuno, equino, ovino, caprino y porcino, cuyo asentamiento complementó en el espacio rural y sustituyó en el urbano las funciones que tuvieron sus antecesoras, las cuales fueron desplazadas a un segundo plano (Castro, 1999, pp. 12-14).

La llegada y establecimiento de especies foráneas traídas por los invasores transfirieron por un lado nuevos usos en la movilidad, transporte de carga, fuerza de trabajo en la agricultura, alimento, materia prima (cuero, lana, sebo, etc.), comercio, intercambio, y recientemente, productos dirigidos al sector turístico, y por otro, nuevas costumbres, como las corridas de toros, la marcación de nuevas especies de animales, la doma de potros, la carrera de caballos, el laceo de reses bravas, entre otras, que con el paso del tiempo fueron asimiladas, resignificadas y arraigadas, generando un profundo sentido de identidad sociocultural local expresado en el ámbito cotidiano con la acepción qorilazo, palabra quechua que significa «lazo de oro» (Villena, 1987).

Hoy, el ganado vacuno mantiene incólume su importancia en todos los ámbitos de la vida diaria y festiva del poblador chumbivilcano. Dicho protagonismo se hace evidente en la mayoría de sus fiestas patronales y en las celebraciones de los aniversarios de creación política de la provincia y sus distritos, donde el turupukllay o corrida de toros costumbrista ${ }^{1}$ es la actividad lúdica por antonomasia.

Actualmente, en el distrito de Santo Tomás, el turupukllay o corrida de toros costumbrista se lleva a cabo en la festividad de Cruz Velakuy el 3 de mayo, en el marco del aniversario de la provincia de Chumbivilcas en el mes de junio, y en el último día de la Fiesta de la Virgen de 
Del mismo modo que sucedió con muchos usos y costumbres foráneas, en Chumbivilcas las corridas de toros fueron transmutadas. Su práctica no admite que se hiera, torture ni asesine al turu, que, junto con el caballo, tiene para la cosmovisión del poblador chumbivilcano un lugar protagónico en su mitología, simbología, vida cotidiana y festiva, claramente visible en los profundos vínculos afectivos que se observan durante las t'inkanas, festividades pecuarias de fertilidad y reproducción donde se les dedica un repertorio musical conocido como wakataki; markanas, ceremonias de marcación donde se les adorna, conversa, canta y baila, así como en el turupukllay, donde se ejecuta un instrumento musical para alegrarlos y/o embravecerlos, el wakawaqra, y un repertorio especial denominado turutaki que les dedica la comunidad, músicos de wakawaqra, cantoras y la banda típica (Villena, 1987).

\section{BANDA TÍPICA}

Conformada instrumentalmente por cornetas, pitos $^{2}$, tambores, bombo y wakawaqras, la banda típica de Chumbivilcas admitió instrumentos musicales castrenses asentados como tales en Europa desde el siglo XV (Latham, 2008, p. 955) que se popularizaron en el territorio peruano desde el siglo XIX (Robles, 2000, p. 69). En tal sentido, cornetas, tambores y pitos son adaptaciones locales provenientes de las cornetas, pífanos, flautas traveseras y tambores militares aunados a instrumentos musicales creados o recreados a partir del encuentro entre la cultura local y la foránea, como el wakawaqra y el bombo (Romero, 2008).

No obstante, en la región andina precolombina ya existían instrumentos de cualidades sonoras harto parecidas, aunque fabricados con otros materiales, tocados en distintos contextos y ejecutados con técnicas diferentes. Así lo evidencian las trompetas naturales, como la trompeta de caracol, pututu o trompetas de cerámica de las culturas Recuay, Pucara o Mochica (Museo Nacional de Antropología y Arqueología, 1981); vestigios de flautas traveseras como la hallada en Atacama (Díaz, 2013, pp. 12-41); indicios de su práctica, como demuestra una escultura de la cultura Chimú (Bolaños, 1985, pp. 36-37) o los distintos tipos de membranófonos de origen precolombino hoy en día vigentes (Instituto Nacional de Cultura, 1978).

la Natividad en setiembre. La corrida costumbrista realizada antiguamente en el mes de julio por el Día de la Independencia del Perú dejó de llevarse a cabo hace varias décadas.

2 Flauta travesera hecha de caña o tubo de plástico, denominada en otras zonas del departamento de Cuzco con el nombre de kinray pito. 
Del mismo modo que sucedió en otras zonas del Perú, como el valle Yanamarca (Romero, 1985, p. 250) y el valle del Mantaro (Romero, 1998, pp. 44-45), en el departamento de Junín, en el valle del Colca, departamento de Arequipa (Ráez, 1998, pp. 284-285; 2002, pp. 26-27) y en las provincias de Bolognesi y Ocros, zona sur del departamento de Ancash (Robles, 2000), es deducible que en la provincia de Chumbivilcas dichos instrumentos también fueran paulatinamente adoptados por las poblaciones originarias que participaron en el servicio militar desde la independencia del Perú, por voluntad propia o compelidas por los hacendados, como sucedió en la provincia de Chumbivilcas durante la Guerra con Chile (Montes, 2008, pp. 333-337).

Después, continuaron su aprendizaje y práctica musical durante su asimilación voluntaria a la vida castrense, buscando algún tipo de movilidad social en la rígida estructura del sistema de hacienda (Villena, 1987, p. 70), y posteriormente en las bandas de música o bandas de guerra de las instituciones educativas de la provincia (Ráez, 1998).

Así, por una parte, los instrumentos musicales militares y su técnica de ejecución fueron asimilados, renovados y unificados al acervo musical vernáculo, constituyendo una nueva sonoridad fuera de su espacio inicial, a través de la transmisión de conocimientos musicales de generación en generación que se dio - $\mathrm{y}$ da - durante la interacción de los niños y jóvenes con sus padres, familiares o allegados músicos en el ámbito festivo y cotidiano. Por otra, quedaron como impronta de sus inicios términos musicales castrenses, como toque, diana, caballería, ataque y retirada, que demuestran la ligazón de la banda típica de Chumbivilcas con su pasado militar.

Antes de la Reforma Agraria, la banda típica era un conjunto musical conformado netamente por pobladores que radicaban en el ámbito rural de la provincia. Si bien su formato instrumental era idéntico al actual, los instrumentos musicales que ejecutaban - con excepción de la corneta, que era adquirida en las ferias provinciales de Canchis y Espinar o de comerciantes del departamento de Puno que llegaban a la festividad de la Virgen de la Natividad en el distrito de Santo Tomás, capital de la provincia - eran fabricados por ellos mismos con los recursos que disponían a su alcance, a mano y con el uso de herramientas adaptadas para sus fines específicos. En ese tiempo los mistis o población mestiza que residía en el ámbito urbano se inclinaba por los instrumentos de cuerdas y el acordeón, los cuales eran ejecutados en el ámbito privado de la vida festiva familiar, como cumpleaños, matrimonios y fiestas patronales, entre otros. 


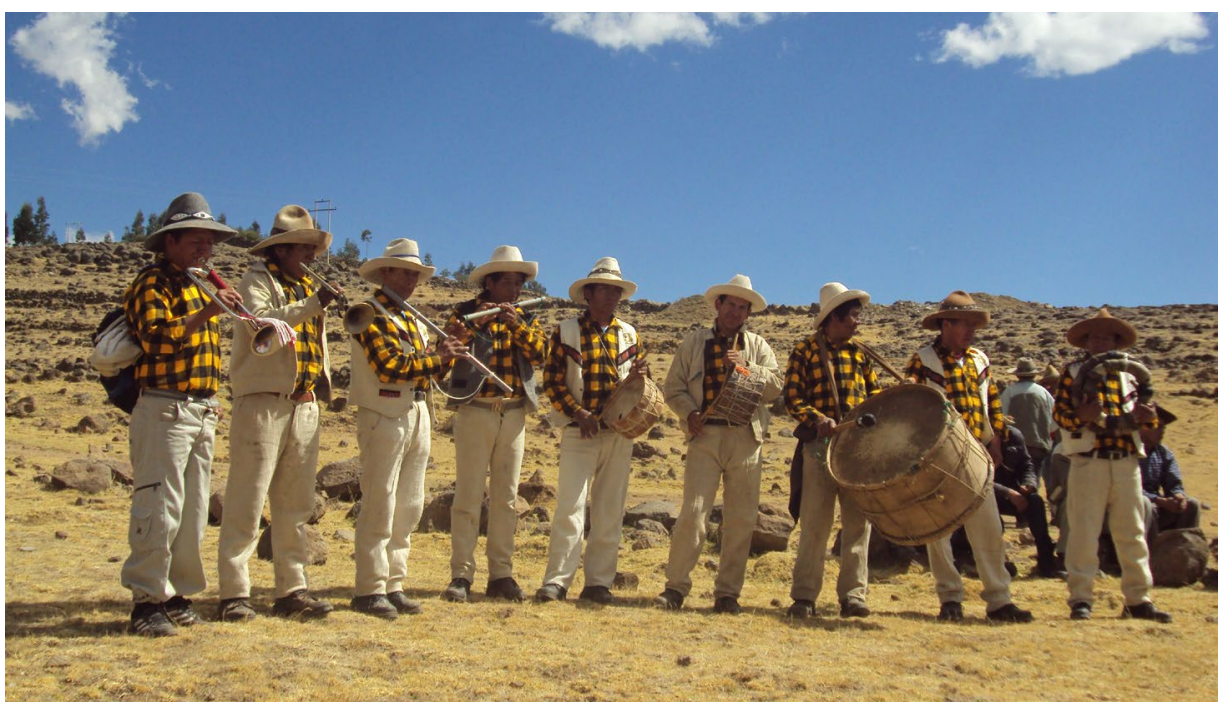

Foto 2. Banda típica Los Auténticos de Marka Marka. Lugar: distrito de Santo Tomás, provincia de Chumbivilcas. Foto: Hubert Ramiro Cárdenas, 2015.

Hoy en día, la flexibilización de la estructura social de la provincia ha permitido el tránsito fluido de los músicos y la población en general entre las comunidades campesinas, centros poblados, distritos y la capital de la provincia, la amplia difusión endoprovincial de bandas típicas y conjuntos de cuerdas por los medios masivos de comunicación, internet, mercados alternativos autogestionados de producción y distribución de música provincial ${ }^{3}$, y la apertura e incremento de un mercado accesible y económico de instrumentos musicales de todo tipo. Actualmente se puede verificar, por un lado, la permanente actualización y fortalecimiento de dichas manifestaciones musicales, y por otro, el tránsito irrestricto de los músicos chumbivilcanos entre bandas típicas y conjuntos de cuerdas en las distintas festividades públicas y privadas de las que participan, evidenciando que la división social, cultural y económica que alguna vez existió en el uso de instrumentos musicales está a punto de extinguirse.

3 El precio del disco compacto de audio (CD-ROM) es de S/ 1, mientras que el de video (DVD), de S/ 2. 


\section{ASPECTOS GENERALES}

\section{Instrumentos musicales}

La banda típica está conformada por instrumentos musicales aerófonos y membranófonos. En el primer grupo se encuentran la corneta - instrumento de viento de metal característico de las bandas de guerra - y el pito, flauta travesera que mide 71 centímetros de longitud, cuenta con seis orificios de digitación y puede ser clasificada de dos maneras: aislada o de un solo tubo cuando está hecha a base de soccos o caña, y en juego cuando está fabricada por dos tubos de plástico ensamblables (Instituto Nacional de Cultura, 1978, pp. 219-220). A su vez, el wakawaqra, corneta de cacho que tiene forma de espiral y mide aproximadamente 220 centímetros de longitud, está conformado por una cantidad variable de trozos de cuernos de ganado vacuno que, dependiendo de su tamaño, oscila entre quince y veinticinco. Dichas piezas están unidas entre sí con brea y estaquillas de madera, forradas con tiras de cuero de vacuno.

En el segundo grupo se encuentran el tambor y el bombo. Están hechos con maderas que se pueden hallar en el ámbito local, hebras de procedencia bovina o sintética, y dos parches de cuero de chivo que se tiemplan mediante un sistema de ajuste de aros de madera y cuerdas. Cuando son fabricados por los mismos músicos, sus medidas son variables. El tambor mide aproximadamente 25 centímetros de diámetro y 15 de profundidad, mientras que el bombo, 65 centímetros de diámetro y 40 de profundidad. El tambor es percutido con dos baquetas sobre el parche superior y el bombo con un mazo cuyo extremo protuberante está formado con textiles. Únicamente el parche inferior del tambor es atravesado por bordones.

Recientemente - aunque en menor medida—, los músicos de las bandas típicas continúan fabricando sus instrumentos musicales; de ahí que, exceptuando la usual adquisición comercial de las cornetas y la fabricación de los wakawaqras, en los últimos años los músicos optan mayoritariamente por utilizar en el resto de instrumentos otra materia prima o por adquirir instrumentos musicales confeccionados con otros materiales o producidos industrialmente. Es el caso de los pitos, los cuales últimamente son fabricados por los músicos con tubos rígidos de plástico que incluso son hechos de dos piezas acoplables; en lugar de los tambores, adquieren redoblantes metálicos con parches de plástico y bombos que son fabricados con placas de madera contrachapada con parches de cuero de chivo o de metal con parches de plástico, al igual que los redoblantes. Asimismo, se observa el paulatino ingreso de la trompeta como instrumento alternativo a la corneta, 
pues, según los músicos, otorga mayores recursos para el acompañamiento de los distintos géneros musicales que interpretan.

Estos instrumentos musicales, antes de ser ejecutados, son preparados por los músicos de la banda típica de distintas maneras. Así, el interior de cornetas, pitos y wakawaqras es levemente lavado con agua para que «tengan voz». Esta acción también sirve para limpiar los instrumentos del polvo e insectos que hayan podido introducirse durante el tiempo que permanecieron guardados. Por otro lado, para que no pierdan consistencia, los parches de los tambores y el bombo son tensados poco antes de comenzar la fiesta y destensados al finalizar. Asimismo, durante la fiesta, son tensados cuando baja la temperatura y destensados cuando sube. Adicionalmente, las trompetas, utilizadas juntamente o en lugar de las cornetas, se limpian con agua tibia, dado que, en las épocas más frías del año, los pistones se traban con el aceite utilizado anteriormente para lubricarlos.

\section{Participación}

La participación de la banda típica está circunscrita tradicionalmente a la animación integral de fiestas patronales de comunidades campesinas y distritos de la provincia de Chumbivilcas. Hoy en día, dichas fiestas tienen dos modalidades según su organización: por una parte, las organizadas por el carguyuq alferado - llamado también albero - , quien se encargará primordialmente dos actividades: la velada y el día central. Estas contarán, sobre todo, con la presencia de los familiares y allegados más cercanos a los organizadores, constituyéndose así en un cargo de carácter familiar. Por otra parte, están las organizadas por el carguyuq torero, que se caracterizan por llevar cabo un programa variable de actividades festivas, de amplia duración y masiva participación, en el marco de la fiesta patronal. En ese sentido, el carguyuq torero realiza varios meses antes de la fiesta patronal, junto con sus familiares y allegados, la llant'ana o tala de árboles que servirán como leña durante la festividad, y alrededor de una semana antes del día central, la aqhana o preparación de la chicha con la que brindarán durante los días de fiesta. Asimismo, días antes del día central, el turu nak'ana, que es el sacrificio de un novillo con cuya carne se prepararán los alimentos del cargo; la banda tiyachina o recepción de los músicos de la banda típica por el carguyuq torero; después, la haykuna o entrada, actividad que consiste en recorrer en centro poblado, junto con una gran comitiva, anunciando que el cargo ha comenzado; el día central de la fiesta patronal, fecha en la cual se realiza el traspaso oficial del cargo; el turu kacharpari, actividad rememorativa en la cual 
se despide a las personas que traerán a los toros para el juego; el toril velana, realizado la víspera del turupukllay, en donde un paqu se encarga de dar una ofrenda a la Pachamama para que el día siguiente el juego de los toros sea exitoso; la tranquilla watana o atado de la tranquilla que facilita el ingreso y salida de los toros al ruedo; el turupukllay, y la banda kacharpari, almuerzo con que los «dueños del cargo» despiden a los músicos de la banda típica.

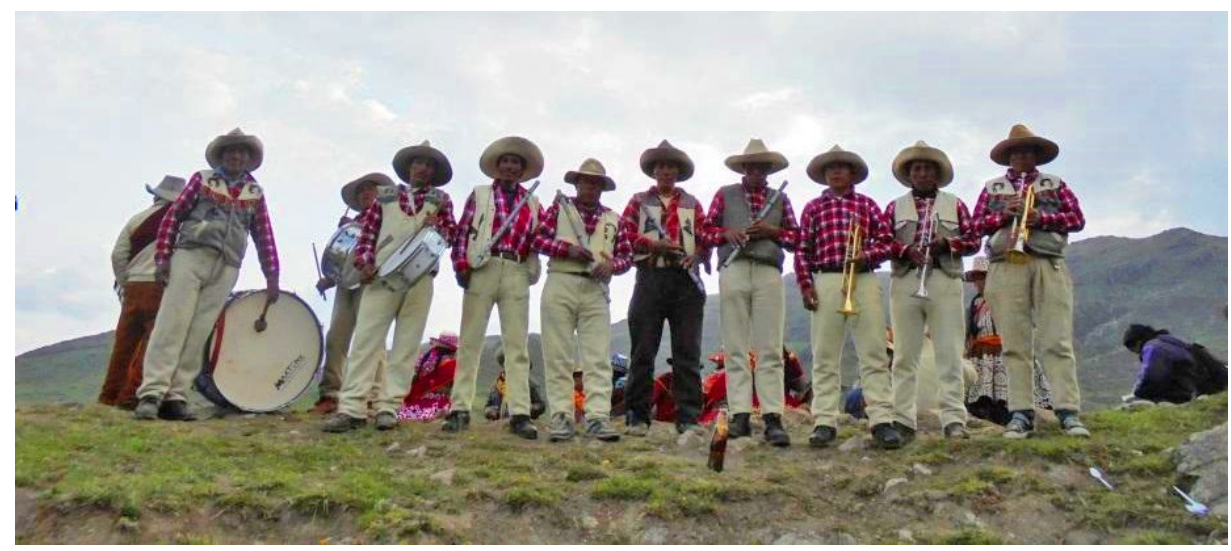

Foto 3. Banda típica Huamanripaq. Lugar: comunidad campesina de Pfuisa, distrito de Llusco, provincia de Chumbivilcas. Foto: Hubert Ramiro Cárdenas, 2018.

En dichas ocasiones, las bandas típicas participan con dos conformaciones instrumentales diferentes, según sea el caso. Así, cuando la fiesta patronal está organizada por el carguyuq alferado, la banda estará conformada por dos músicos de corneta, dos de pito, dos de tambor y uno de bombo. Dado el carácter esencialmente familiar de este cargo, en ocasiones no se cuenta con la participación de una banda típica; por tal motivo, la fiesta se realiza únicamente con música popular local y nacional emitida mediante un equipo de sonido.

Si la fiesta patronal está organizada por el carguyuq torero, la banda estará conformada por dos músicos de corneta, dos de pito, dos de tambor, uno de bombo y dos de wakawaqra. La presencia de los wakawaqreros es imprescindible durante la bandatiachina, haykuna, torocacharpari, toril velana, tranquilla watana y el turupukllay, no obstante, si el carguyuq lo solicita, pueden participan sin inconvenientes en todas las actividades de la fiesta patronal.

En las últimas décadas, también son contratados ocasionalmente para animar corridas de toros costumbristas realizadas en las provincias de Cuzco, Espinar y Paruro, en el departamento de Cuzco y algunos poblados, aledaños a la provincia 
de Chumbivilcas, de los departamentos de Apurímac y Arequipa. Del mismo modo, desde el año 2014, son invitados por la Dirección Desconcentrada de Cultura de Cusco, Ministerio de Cultura del Perú, a participar en actividades de difusión musical provincial en las capitales del departamento de Cuzco y la provincia de Chumbivilcas.

Asimismo, dicho conjunto ha conseguido integrarse exitosamente a otros tipos de actividades, como celebraciones de matrimonios; breves intervenciones, a manera de despedida, en funerales de músicos o personas que se deleitaron con la banda típica; serenatas de aniversarios de los distritos y la provincia, los cuales son organizados por los gobiernos locales y festejados suntuosamente en sus plazas mayores, y eventos costumbristas de asociaciones de residentes chumbivilcanos en otras provincias de $\mathrm{Cuzco} u$ otros departamentos colindantes.

En este tipo de eventos, frecuentemente realizados en lugares amplios y dirigidos a un público multitudinario, las bandas típicas participan libremente con una de sus dos conformaciones instrumentales, utilizando sin ningún inconveniente consolas de sonido, micrófonos y altoparlantes para amplificar el sonido de sus instrumentos musicales y, en ocasiones, las voces de cantoras que acompañan a la agrupación.

\section{Conformación instrumental}

La banda típica de Chumbivilcas generalmente está conformada por nueve integrantes, autodenominados componentes, quienes durante la ejecución se ubican de izquierda a derecha manteniendo el estricto orden instrumental de dos ejecutantes de corneta, dos de pito, dos de tambor, uno de bombo y dos de wakawaqra. A menudo, los instrumentistas de la banda típica asumen el nombre del instrumento que ejecutan o se llaman entre ellos como cornetero, pitero, tamborero, bombero y wakawaqrero, waqrero o simplemente waqra.

Dicha conformación se manifiesta de dos maneras: la primera está constituida por los nueve integrantes mencionados, mientras que la segunda cuenta con siete: dos ejecutantes de corneta, dos de pito, dos de tambor y uno de bombo. Los dos músicos de wakawaqra ${ }^{4}$ faltantes son contratados por separado para aunarse a la banda durante el cargo o celebración.

4 En la provincia de Chumbivilcas, los fabricantes y músicos de wakawaqra provienen primordialmente de los distritos de Llusco y Quiñota. 


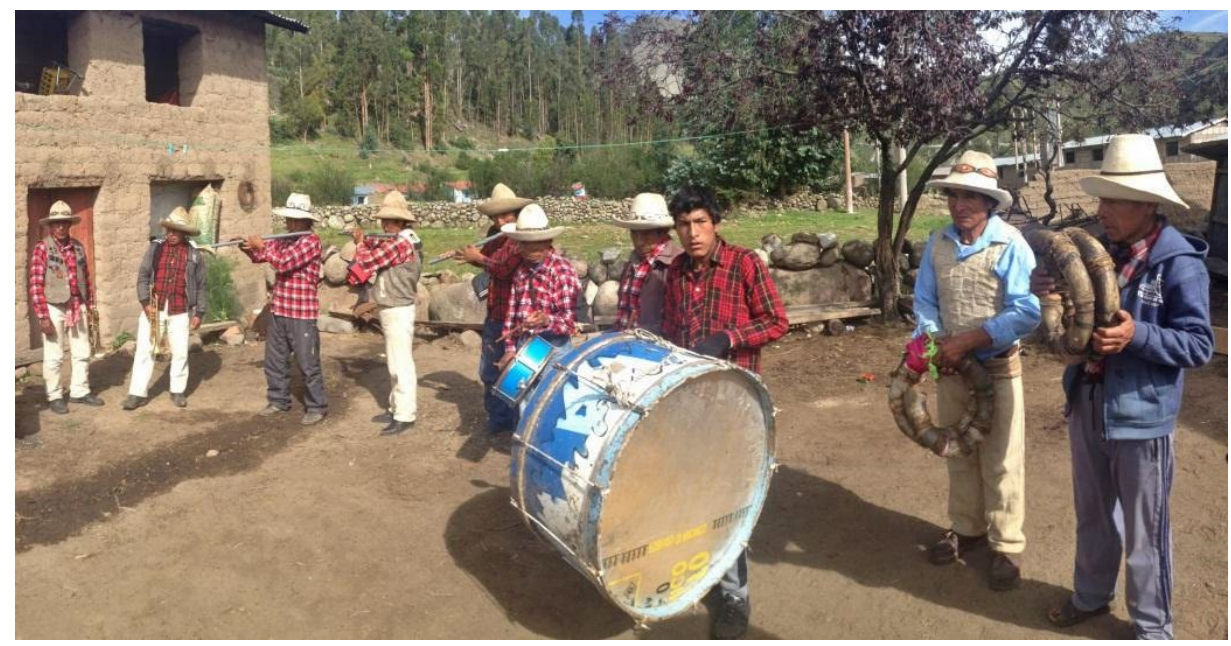

Foto 4. Banda típica Santo Tomás acompañada por waqras de la comunidad campesina Pallpa Pallpa, distrito de Quiñota. Lugar: comunidad campesina de Kututo, distrito de Llusco, provincia de Chumbivilcas. Foto: Hubert Ramiro Cárdenas, 2018.

La conformación instrumental de la banda es flexible; en ese sentido, existen bandas típicas que tienen una conformación cuyo número de integrantes es superior al habitual. Esta variación se debe a que, en primer lugar, existen bandas integradas por un número mayor de componentes; en segundo lugar, los componentes adicionales son músicos que están en proceso de aprendizaje, y en tercer lugar, son proporcionados como un favor de amistad y buena voluntad por los familiares o allegados del cargo para que acompañen a la «banda oficial». Esta manera peculiar de apoyo, otorgada en bienes o servicios, es denominada: wikch'upa.

Por otro lado, el número de integrantes de la banda típica, como tal, no interviene en la apreciación de la población sobre un cargo, pues en el ámbito musical, los buenos o malos comentarios se basan en el prestigio y competencia de banda típica, los músicos o bandas concedidas como wikch upa por los familiares y allegados de los carguyuq, y recientemente, el uso de equipos de amplificación de sonido, la presencia de un animador o animadores y la contratación de orquestas musicales o «conjuntos de cuerdas» que únicamente toman parte de la fiesta patronal en la noche que antecede el día central.

Es oportuno mencionar que en el último lustro se generó un cambio en dicha alineación, pues a la formación acostumbrada de la banda típica - tradicionalmente integrada por varones-, se incorporó a una o dos cantoras, quienes 
se encargaban de cantar los temas que otrora eran interpretados por la colectividad durante las festividades ${ }^{5}$.

\section{Organización de la banda típica}

La organización interna de la banda está íntimamente relacionada con el chas$k i c h a s k i$, expresión que indica el modo de ejecución complementario en el cual uno de los dos ejecutantes de corneta, pito y wakawaqra, sobre la base rítmica de los tambores y bombo, inician su respectiva melodía, mientras que sus pares se aúnan recibiendo, prolongando y complementando la frase, permitiendo que su símil tenga el tiempo necesario para respirar e iniciar la siguiente, lo que posibilita la continuidad fluida de la interpretación. La lógica del chaskichaski también es practicada y validada por los músicos de tambor y las cantoras ${ }^{6}$.

\section{Gráfico 1. Representación gráfica del chaskichaski}

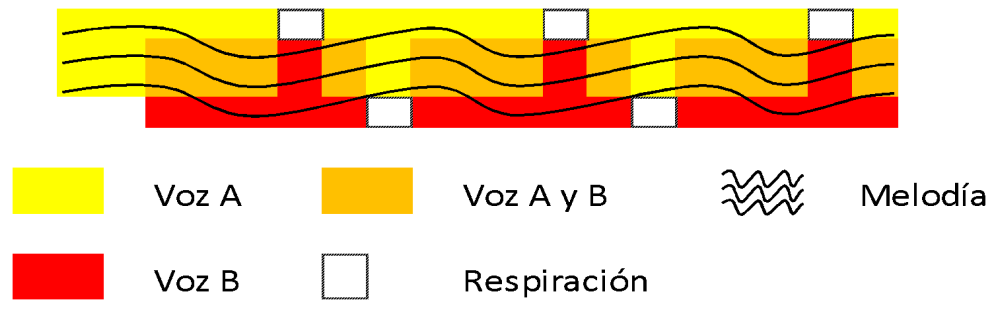

En tal sentido, para que una parte de los instrumentos musicales pueda iniciar y la otra recibir, prolongar y complementar el sonido, deben tener las mismas voces. Así, en el caso de los wakawaqras —instrumento constituido por la unión de varios trozos de cuernos de bobino, correspondientes al mismo lado, a manera de espiral—, si no fueron construidos en pareja, se les agregan o restan una o varias piezas hasta igualar su sonido ${ }^{7}$. En el caso del pito, dado que tiene un tapón

5 Esta innovación tuvo gran aceptación desde su aparición en el año 2014 hasta mediados de 2017, fecha en que los componentes de la banda típica que promovía este cambio, Llaqtay Fiesta, decidieron tomar un descanso por motivos personales. En tal sentido, el proceso de consolidación o desintegración de dicha innovación aún está en marcha.

6 Escuchar audio 1. Chaskichaski - Interpretación de turuwaynu con wakawaqras. http://revistas.pucp.edu.pe/imagenes/anthropologica/01_Chaskichaski.mp3

7 Existen datos que manifiestan que los wakawaqras de sonido agudo son llamados lambrameños, y los de sonido grave, $q$ 'osñiwakawaqra (Instituto Nacional de Cultura, 1978, p. 265; Josafat Roel, citado por García, 1978, pp. 3-19). No obstante, en el trabajo de campo realizado entre 
en la parte superior del orificio de insuflación, este es empujado hacia adentro para que el sonido sea más agudo y hacia afuera para que sea más grave, según su par. Con relación a los tambores, ambos deben ser del mismo material, es decir, fabricados a mano, con madera y cuero de chivo o de procedencia industrial, de metal con parches de plástico. Respecto de las cornetas, los músicos consideran que tienen una sonoridad estándar.

Al mismo tiempo, según el rol que asume cada componente durante la ejecución en chaskichaski, se reconocen como corneta mayor y segunda corneta, primer y segundo pito, primer y segundo tambor, primer y segundo wakawaqra ${ }^{8}$ o lluq'iwakawaqra y pañawakawaqra, donde los primeros, debido a su experiencia y destreza en la ejecución de varios instrumentos, o cantando, así como el conocimiento de un vasto repertorio, guían la ejecución, y los segundos, músicos quienes en algunos casos aún están en proceso de aprendizaje, se desenvuelven como soporte y complemento musical de sus congéneres sobre la base rítmica del bombo. Cuando se incorpora uno o más instrumentos a los dúos, se ubican asumiendo el orden correlativo establecido o integran un nuevo dúo -o incluso trío-, el cual participa intercaladamente con el «oficial».

Además de los roles mencionados, uno de los componentes tiene responsabilidades adicionales al resto de la banda. Es el cabecilla, cabeza o uma, integrante elegido por los mismos músicos para representar al conjunto en la gestión de los contratos con las personas e instituciones interesadas en el servicio de la banda. Como músico, el uma debe ser versátil y experto en la ejecución de varios instrumentos y capaz de suplir la ausencia circunstancial de cualquiera de sus compañeros durante el cargo o celebración.

los años 2015 y 2016 en los distritos de Santo Tomás, Llusco y Quiñota, no se logró verificar esta información. Con relación a este punto, se puede constatar que Lambrama es un distrito de la provincia de Abancay, departamento de Apurímac, área donde también se cultiva el peculiar instrumento, y que $q$ 'osñ $i$, color humo o ahumado de algunos vacunos, era el nombre con el que se conocía antiguamente al wakawaqra y, en consecuencia, a su instrumentista.

8 A diferencia de otras «cornetas de cacho» de otros lugares del Perú, cuya pareja está compuesta por hembra y macho según el lado de los cuernos con los que fueron fabricadas (Instituto Nacional de Cultura, 1978, p. 264; Pinto, 1993, p. 27), en la provincia de Chumbivilcas el dúo está formado por lluq'iwakawaqra y pañawakawaqra, es decir, wakawaqra izquierdo y wakawaqra derecho, los cuales hacen también referencia al lado de las astas con los que fueron fabricados. Estos nombres son mayormente utilizados por los músicos en el ámbito rural, donde participan también acompañados por una tinya y voces en t'inkanas y markanas de ganado vacuno realizadas en la provincia. 


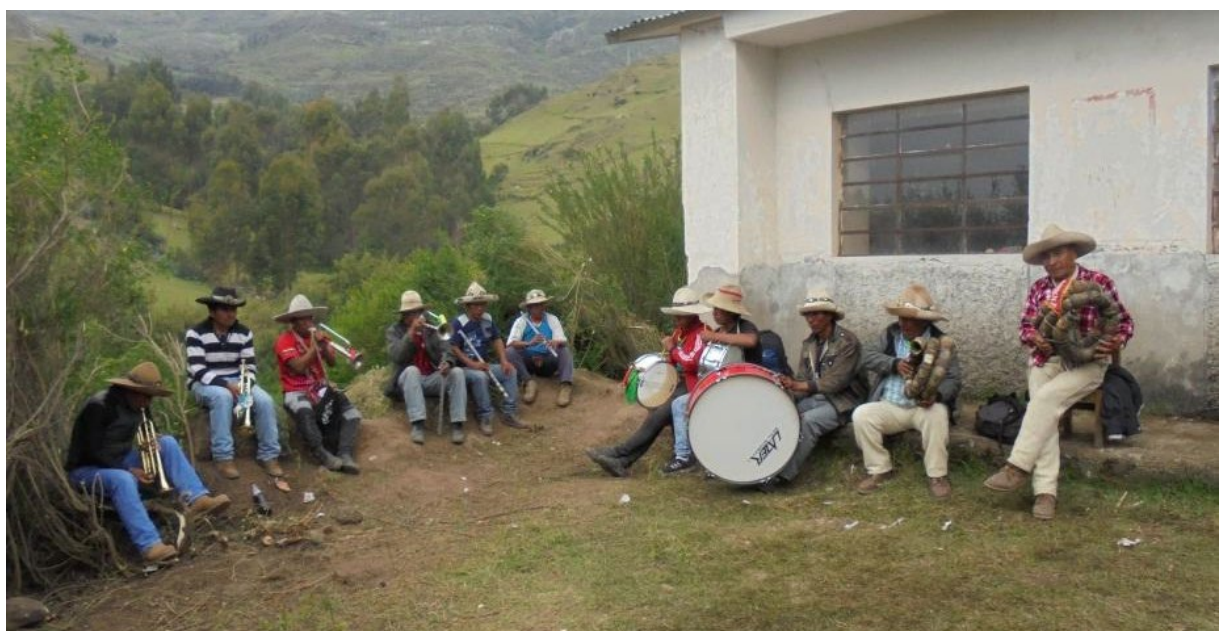

Foto 5. Banda típica Nietos de Taukamarka, acompañados por dos wikch 'upa trompeteros y waqras de la comunidad campesina de Pfuisa, distrito de Llusco. Lugar: comunidad campesina de Pfuisa, distrito de Llusco, provincia de Chumbivilcas. Foto: Hubert Ramiro Cárdenas, 2018.

Los acuerdos pactados — muchas veces realizados únicamente de forma verbal - consisten en el adelanto del cincuenta por ciento de la remuneración económica al iniciar la fiesta, alimentación y alojamiento durante los días que dure el cargo o celebración, y el cincuenta por ciento restante al final. La remuneración económica que recibe cada uno de los componentes de la banda típica, incluyendo el uma, es equitativa y contabilizada por cada día de servicio?.

Una vez que se establece el contrato, el uma tiene la responsabilidad de visitar la casa de cada uno de los componentes para darles a conocer los términos del acuerdo. Asimismo, comunica a los familiares de los músicos que se hace responsable por ellos, garantizando a sus padres o esposas su buena salud, bienestar y justa retribución por el trabajo que realizarán en la fiesta patronal o actividad para la que fueron contratados.

\section{Temática musical}

Durante mucho tiempo, la materia de los temas interpretados por la banda típica está en gran medida vinculada con la vida cotidiana de la provincia de Chumbivilcas, es decir, con la vida campestre de la región, la belleza femenina,

9 A febrero de 2018, la remuneración económica por cada músico fluctúa entre los S/ 60 y S/ 120 por día, importe que varía según la reputación de la banda típica. 
su gente valerosa y sus costumbres ganaderas, al turupukllay, a sus animales compañeros como el caballo y el toro, a sus paisajes de llanuras y pastizales, a la virgen, santa patrona o santo patrón ${ }^{10}$, entre otros.

Como producto de la creciente cobertura de la radio, iniciada desde mediados del siglo XX e intensificada en las décadas posteriores en todo el Perú, su repertorio se fue enriqueciendo con temas populares del huayno comercial y la música chicha. Estos temas, de procedencia tradicional o contemporánea, son adaptados a un formato regular en el que un conjunto de temas son interpretados consecutivamente ${ }^{11}$.

Durante la ejecución ininterrumpida de este conjunto de temas, las cornetas tienen un papel notable, pues además de iniciar cada tema, intermediar entre los temas ejecutados llamando al siguiente y dar el alto para concluir su intervención y descansar, indican con su toque el tema que será interpretado ${ }^{12}$.

Por otra parte, los temas interpretados bajo el formato musical de la banda típica disponen que la corneta mayor, complementada por la segunda corneta, inicien el tema ${ }^{13}$; luego se unen los tambores y el bombo, dando paso al primer y segundo pito, cuyo ingreso se empalma con las cornetas justamente durante la parte final de su intervención. Los wakawaqras intervienen momentáneamente, por lo general en la primera parte de cada tema musical, acompañando a los pitos, tambores y bombo. No obstante, también toman parte poco antes del llamado de las cornetas, con las que muchas veces se empalman sonoramente.

Asimismo, cuando el resto de la banda está descansando, tocan en dueto tonadas instrumentales o acompañan los cantos de las mujeres presentes en la festividad. Estos cánticos forman parte del turutaki, repertorio musical conformado por cantos femeninos sin acompañamiento de instrumentos musicales, cantos acompañados por wakawaqras o la banda típica, o temas netamente instrumentales dedicados a los actores del turupukllay y ejecutados sobre todo en el marco de la corrida costumbrista ${ }^{14}$.

10 Los temas dedicados a la virgen, santa patrona o santo patrón son exclusivos y denominados como «alabado». Escuchar audio número 2. Toque de alabado.

http://revistas.pucp.edu.pe/imagenes/anthropologica/02_Toque_de_alabado.mp3

11 El tiempo del conjunto de temas musicales oscila aproximadamente entre los quince y veinticinco minutos.

12 Escuchar audio número 3. Enlace entre dos temas de un mismo bloque musical por cornetas Toque waynu.

http://revistas.pucp.edu.pe/imagenes/anthropologica/03_Muestra_de_Waynu.mp3

13 Escuchar audio número 4. Llamada de cornetas - Toque turumarinera. http://revistas.pucp.edu.pe/imagenes/anthropologica/04_Muestra_de_Turumarinera.mp3

14 Escuchar audio número 5. Toque Turuwaynu - Interpretación con wakawaqras y voces. $\mathrm{http} / / /$ revistas.pucp.edu.pe/imagenes/anthropologica/05_Toque_turuwaynu.mp3 


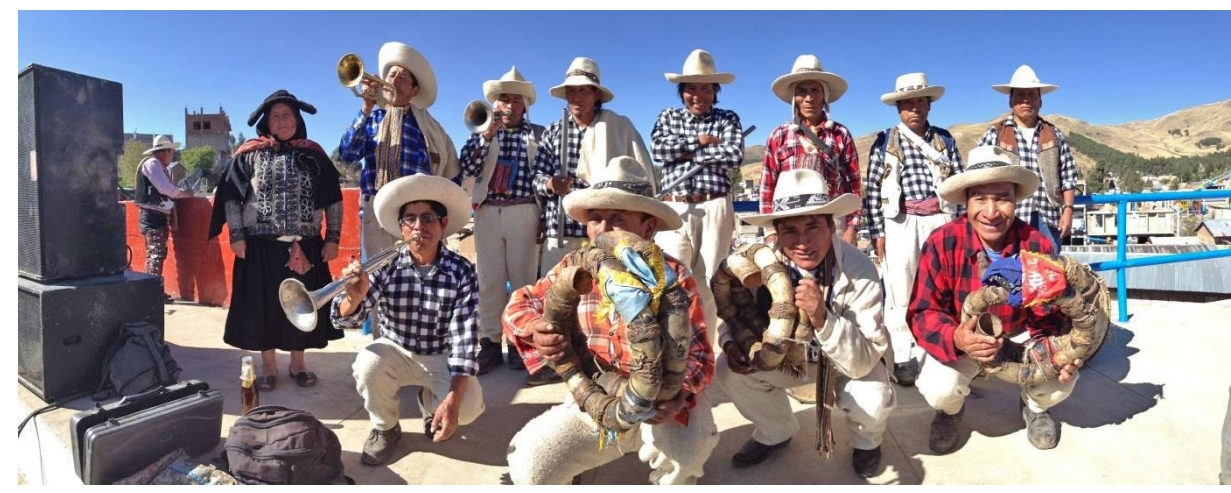

Foto 6. Banda típica Llaqtay Fiesta, acompañada por cantora. Lugar: distrito de Santo Tomás, provincia de Chumbivilcas. Foto: Hubert Ramiro Cárdenas, 2015.

Los cánticos femeninos en solitario son conocidos por los pobladores como wankas. Estos eran interpretados por un grupo de cuatro o seis mujeres durante las wasichanas o techado con paja de las viviendas en las comunidades campesinas y centros poblados; en los trabajos de la siembra durante los meses de agosto, setiembre y octubre en las zonas agrícolas de la provincia de Chumbivilcas; en las fiestas patronales, dedicadas a la virgen, santa o santo patrono de la población durante la noche de víspera del día central y otras partes de la fiesta; en las batallas rituales que se realizan en Toqto - zona fronteriza ubicada entre las provincias de Canas y Chumbivilcas - y aquellas que algunas personas de mayor edad recuerdan haber oído en su niñez, y las wankas de despedida, dedicadas a los familiares que realizaban algún viaje lejos de su hogar. Es posible que estas últimas fueran olvidadas casi por completo, pues además de no escucharse hace varias décadas, las personas adultas y de menor edad del distrito no saben de su existencia.

Adicionalmente a estas turuwankas, wankas agrícolas, wasichana wankas, santo wankas - dedicadas a la santa patrona o santo patrón- y las wankas guerreras, están las urpi wankas, cantadas para la pedida de mano de la futura esposa (Montes, 2016). Dada la gran variedad de tipos de wankas existentes y desaparecidas de la provincia de Chumbivilcas, quizás algunas sin mencionar en estas líneas, es altamente probable que sus distintas clases sean conocidas en distinta medida según las zonas geográficas donde eran y son practicadas. 


\section{Toques característicos}

Los temas del repertorio de la banda típica son clasificados por sus componentes en toques, los cuales están formados por conjuntos o bloques de temas musicales que son iniciados, intermediados y finalizados por la corneta mayor y la segunda corneta, en determinadas partes de la fiesta patronal. Los toques más utilizados son la diana, que es tocada a la primera hora del día para iniciar las actividades y también cuando la comitiva del cargo llega a algún lugar, antes de descansar. La diana que toca la banda típica es un bloque musical compuesto por tres temas musicales ${ }^{15}$.

Los toques de pasacalle no son bailables: se ejecutan únicamente para acompañar los desplazamientos de los carguyuq y sus invitados cuando van de un lugar a otro durante la fiesta patronal. Se dividen en dos: el toque de caballería o para caballos, el cual se toca cuando la comitiva está acompañada por jinetes montados; cuando la comitiva parte de un lugar para movilizarse a otro se le llama toque de retirada, y cuando sale el toro al ruedo o ingresan los caballos al turupukllay se denomina toque de ataque ${ }^{16}$.

El toque de pasacalle para peatones se ejecuta cuando la comitiva en movimiento está únicamente integrada por personas. Los toques de pasacalle, dado el papel que tienen en el desplazamiento de la delegación del cargo, llegan a ser bastante prolongados; no obstante, como el resto de toques, siempre son dirigidos por las cornetas ${ }^{17}$.

Además, se tienen también los toques de turumarinera y turuwaynu, y los toques de waynumarinera y waynu. La diferencia de estos toques de símil nombre es correlativa al motivo de la festividad en la que son interpretados. Así, turumarinera y turuwaynu ${ }^{18}$, junto con la música de los wakawaqras, acompañarán al carguyuq torero en todas las actividades del cargo en general, y sobre todo en el turupukllay de fin de fiesta, donde integrarán el turutaki, mientras que waynumarinera y waynu ${ }^{19}$ tendrán lugar en festividades donde no se lleve a cabo

15 Escuchar audio número 6. Toque diana.

http://revistas.pucp.edu.pe/imagenes/anthropologica/06_Toque_Diana.mp3

16 Escuchar audio número 7. Toque pasacalle para caballos - Ataque - Retirada.

http://revistas.pucp.edu.pe/imagenes/anthropologica/07_Toque_Pasacalle_para_Caballos.mp3

17 Escuchar audio número 8. Toque pasacalle para personas.

http://revistas.pucp.edu.pe/imagenes/anthropologica/08_Toque_Pasacalle_para_Personas.mp3

18 Escuchar audio número 9. Toque turumarinera y número 10. Toque turuwaynu.

http://revistas.pucp.edu.pe/imagenes/anthropologica/09_Toque_Turumarinera.mp3

http://revistas.pucp.edu.pe/imagenes/anthropologica/10_Toque_Turuwaynu.mp3

19 Escuchar audio número 11 . Toque waynumarinera y número $1 \overline{2}$. Toque waynu.

http://revistas.pucp.edu.pe/imagenes/anthropologica/11_Toque_Waynumarinera.mp3

http://revistas.pucp.edu.pe/imagenes/anthropologica/12_Toque_Waynu.mp3 


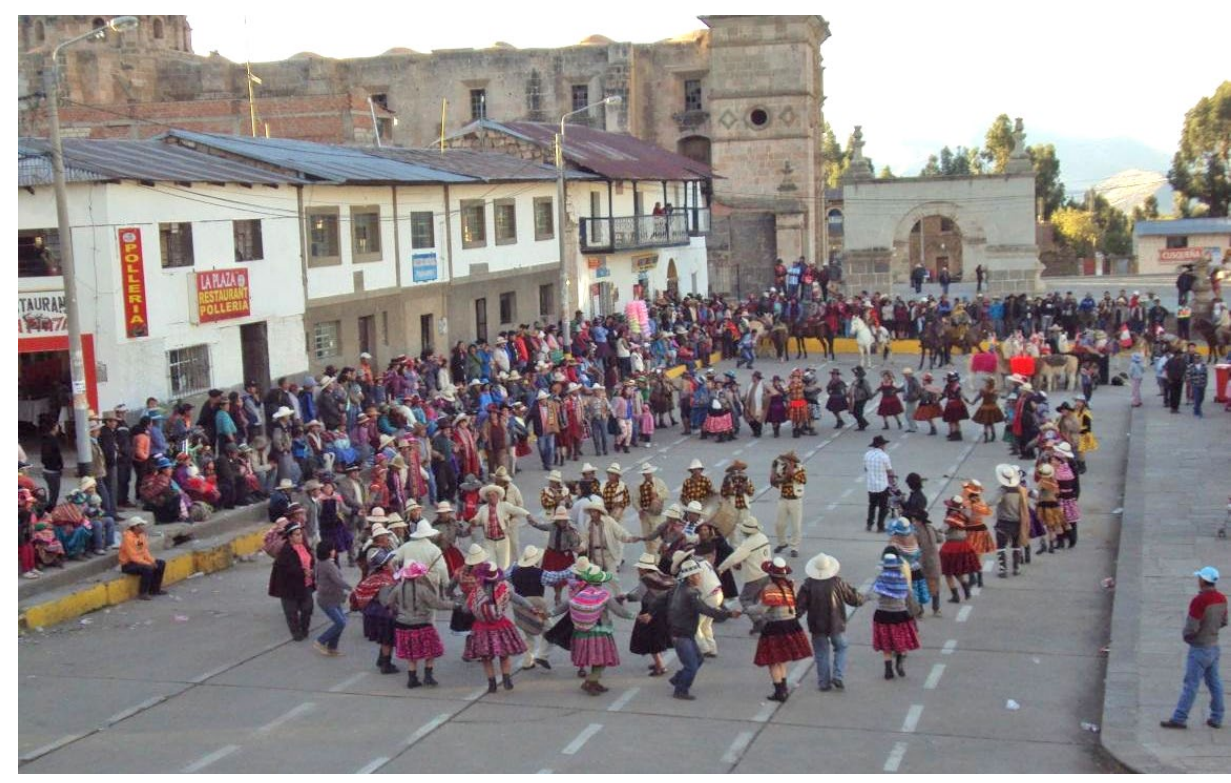

Foto 7. Banda típica en medio de una qaswa, durante la haykuna o entrada, inicio de la fiesta de la Virgen de la Natividad. Lugar: distrito de Santo Tomás, provincia de Chumbivilcas. Foto: Hubert Ramiro Cárdenas, 2015.

la corrida costumbrista. Los waynomarineras y waynus también son interpretados libremente durante la fiesta patronal organizada por el carguyuq torero.

Tradicionalmente, la banda típica de Chumbivilcas interpreta estos toques como un grupo de temas musicales integrados por tres turumarineras o waynumarineras para tocar inmediatamente después alrededor de cuatro turuwaynus o waynus, según sea el caso. A su vez, dichos subgrupos son integrados por un solo tema que se repite o por temas diferentes ejecutados según el número que corresponde a cada toque $e^{20}$, los cuales finalizan opcionalmente con una fuga. Generalmente se incrementa el número de turuwaynus o waynus indeterminadamente, hasta que las cornetas toquen el alto del bloque musical ${ }^{21}$. Por otra parte, cuando están animando el turupukllay, el conjunto de temas es iniciado por el toque de ataque, después del que se ejecuta la secuencia de turumarinera y turuwaynus indicada.

20 Se puede constatar que son las bandas típicas de antes las que tocan reiterativamente solo un tema, mientras que las más recientes integran diferentes en el bloque musical.

21 Escuchar audio número 13. Toque de alto. http://revistas.pucp.edu.pe/imagenes/anthropologica/13_Toque_de_Alto.mp3 
Gráfico 2. Ejemplificación de los toques en bloques musicales

\begin{tabular}{|c|c|c|c|c|c|c|c|c|c|c|c|c|c|c|c|c|c|c|c|c|c|}
\hline \multirow{3}{*}{$\mathrm{Ll}$} & \multicolumn{8}{|c|}{ Turumarinera / Waynumarinera } & & \multicolumn{11}{|c|}{ Turuwaynu / Waynu } & \\
\hline & \multirow{2}{*}{1} & \multirow{2}{*}{\begin{tabular}{|l|}
$\mathrm{TM}$ \\
$\mathrm{WM}$
\end{tabular}} & \multirow{2}{*}{$\mathrm{Ll}$} & \multirow{2}{*}{2} & \multirow{2}{*}{$\frac{\mathrm{TM}}{\mathrm{WM}}$} & \multirow{2}{*}{$\mathrm{Ll}$} & \multirow{2}{*}{3} & \multirow{2}{*}{\begin{tabular}{|l|} 
TM \\
WM
\end{tabular}} & \multirow[t]{2}{*}{$\mathrm{Ll}$} & \multirow{2}{*}{1} & TW & $\mathrm{Ll}$ & \multirow{2}{*}{2} & \multirow{2}{*}{\begin{tabular}{|c|}
$\mathrm{TW}$ \\
$\mathrm{W}$
\end{tabular}} & \multirow{2}{*}{$\begin{array}{l}\mathrm{Ll} \\
\mathrm{L} 1\end{array}$} & \multirow{2}{*}{3} & \multirow{2}{*}{\begin{tabular}{|c|}
$\mathrm{TW}$ \\
$\mathrm{W}$
\end{tabular}} & \multirow{2}{*}{$\frac{\mathrm{L} 1}{\mathrm{~L} 1}$} & \multirow{2}{*}{4} & TW & \multirow[t]{2}{*}{$\mathrm{Al}$} \\
\hline & & & & & & & & & & & W & $\mathrm{Ll}$ & & & & & & & & $\mathrm{W}$ & \\
\hline $\mathrm{Ll}$ & & Llam & & & & & & & & & & WM & & Was & um & & & & & & \\
\hline TM & & Turun & mari & & & & & & & & & W & & Wayl & & & & & & & \\
\hline TW & & Turux & wayı & & & & & & & & & $\mathrm{Al}$ & & Toqu & e de & Alto & & & & & \\
\hline
\end{tabular}

Las canciones de huayno comercial, música chicha, y otros géneros musicales populares modernos, además de ser transmutadas al formato musical de la banda típica, son indefectiblemente adaptadas al toque de waynu. Asimismo, si el carguyuq lo desea, puede pedir anticipadamente al uma que la banda típica prepare para la festividad temas de géneros musicales específicos, como por ejemplo wayliyas, diabladas o waylas.

Asimismo, cuando finaliza el turupukllay, la banda típica interpreta el tema de kacharpari o despedida, Chikchischay paraschay ${ }^{22}$, con el que los invitados de los carguyuq, sus allegados y el público presente, ingresan al ruedo tomados de la mano bailando en círculo - es decir, qhaswando - y rodeando a la banda típica, dando fin a la fiesta del día.

Actualmente, durante la fiesta patronal, el bloque musical waynumarinerawaynu es interpretado con menor frecuencia que antes; en su lugar, se interpreta un amplio bloque musical de waynu, huayno comercial o música chicha. No obstante, dicho repertorio tradicional es interpretado cuando lo solicitan los carguyuq o invitados del cargo. Por otra parte, el bloque turumarinera-turuwaynu y el tema de kacharpari, Chikchischay paraschay, ambos interpretados exclusivamente en el marco del turupukllay, mantienen intacto su vigor.

\section{Vestido}

Su vestimenta característica está confeccionada principalmente de lana de oveja. Las prendas que componen el traje de los músicos son el sombrero, birrete o chullo; camisas a cuadros de lanilla que, según sus colores, sirven para identificar a las bandas típicas en el ámbito local; chaleco de bayeta doble que puede utilizarse por ambos lados y sobre cuya espalda se borda el nombre de la agrupación;

22 Escuchar audio número 14. Chikchischay paraschay - Tema de kacharpari o despedida. http://revistas.pucp.edu.pe/imagenes/anthropologica/14_Chikchischay_paraschay.mp3 
casaca de bayeta con botones o broches; chalina; pantalón de bayeta doble que puede utilizarse por ambos lados; chumpi o faja para sujetar el pantalón; ch 'uspa para llevar hojas de coca, llipt'a y cigarrillos; ojotas o zapatos de cuero, y poncho que, además de ser utilizado por la noche como abrigo, sirve para cubrir los instrumentos musicales cuando llueve.

El traje de las cantoras reivindica la vestimenta tradicional de las mujeres de la población originaria de la provincia. Confeccionado sobre todo de bayeta, está integrado por una montera hecha de paja brava o ch'illwa, recubierta con la tela mencionada de color negro, debajo de la cual usan un velo del mismo material y color al que llaman suk'u o suk'uta; camisa blanca sobre la que visten chaqueta gris, cuyos bordes delanteros llevan cosida una tela negra que en ocasiones lleva zurcido con hilo blanco motivos florales, y una ch 'uspa o bolso pequeño que cruza el pecho, usado para guardar hojas de coca y llipt'a. Para abrigarse, se cubren la espalda y hombros con un phullu o mantón negro encima del que llevan una lliklla o manta en la que transportan productos diversos. Asimismo, usan en la cintura un chumpi o faja con la que sujetan la distintiva pollera negra y, a manera de enaguas, tres o más $p$ 'istus o mantillas, usualmente blancas.

Foto 8. Vestimenta de integrantes de banda típica. Lugar: distrito de Santo Tomás, provincia de Chumbivilcas. Foto: Hubert Ramiro Cárdenas, 2015.

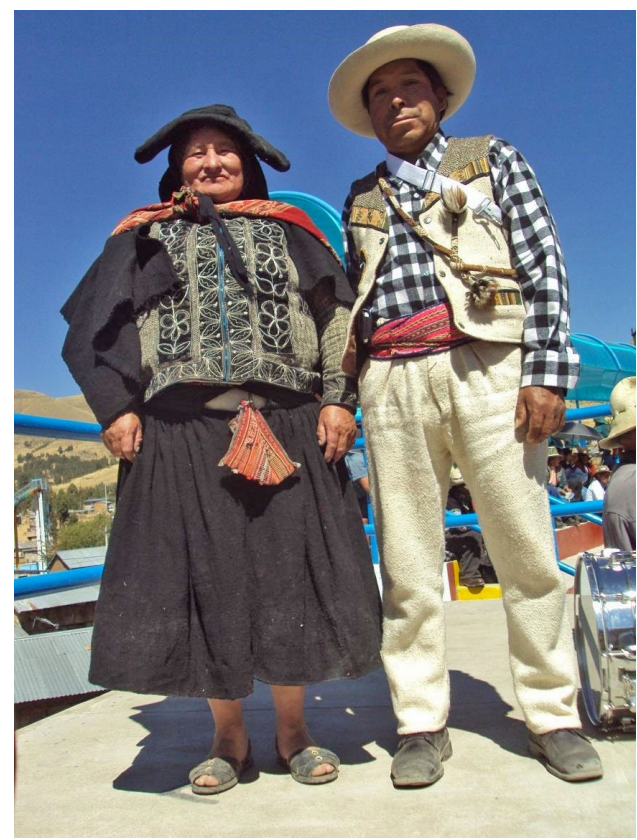




\section{EPÍLOGO}

La banda típica ha logrado adaptarse a los vertiginosos cambios del tiempo, a partir de la estrecha ligazón que guarda con el turupukllay o juego de los toros, corrida de toros costumbrista representativa de la provincia de Chumbivilcas, circunscrita inicialmente a las fiestas patronales y posteriormente incorporada o desdoblada a otras festividades públicas de cobertura provincial, departamental y nacional.

El arraigo de la banda típica a un vigoroso contexto como el turupukllay ha permitido que continúen en la memoria colectiva local características musicales propias, como su formato instrumental, toques particulares y bloques de interpretación, nombres y roles de los músicos, organización de la banda, técnicas de ejecución instrumental, fabricación de instrumentos musicales y el chaskichaski, lógica de ejecución musical complementaria.

Asimismo, la progresiva integración de la provincia de Chumbivilcas al resto del departamento y el país, a partir del proceso de modernización intensificado desde la segunda mitad del siglo XX, permitió que la banda típica se mantuviese abierta a la recepción e interpretación de otras músicas, motivo por el cual enriquecieron su repertorio tradicional, adaptando al formato y musicalidad de la banda típica diferentes géneros musicales, como el huayno comercial, la música chicha, entre otros.

De igual manera, se observa que los músicos de la banda típica han logrado adecuar nuevos materiales para la fabricación de sus instrumentos musicales, promover cambios como integrar cantoras para interpretar las canciones otrora interpretadas colectivamente, utilizar equipos de amplificación para presentarse en lugares con mayor audiencia e incursionar en la producción fonográfica y audiovisual autogestionaria dirigida sobre todo a un público local.

Así, la banda típica se configura como una dinámica agrupación musical tradicional en constante actualización, cuya transmisión oral de conocimientos musicales, fomentada habitualmente en los ámbitos festivo y cotidiano, es complementada con la adopción de medios de comunicación masivos, el acceso y uso de soportes digitales (CD Rom, DVD y USB), así como recursos del internet (redes sociales y videos en línea), utilizados para la difusión, recepción y fortalecimiento de su música en sus propios espacios socioculturales. 


\section{Nota}

El registro de audio y difusión de todas las muestras musicales que acompañan este artículo fueron autorizadas por sus intérpretes, la banda típica Llaqtay Fiesta.

\section{Intérpretes de las muestras musicales}

Las muestras musicales son interpretadas por la banda típica Llaqtay Fiesta, conformada por las cantoras Purificada Montañez Márquez y Nida Layme Sivana; los corneteros, Policarpo Montañez Maqui y Santos Huillca Ancalla; los piteros, Celso Álvarez Sivana, Domingo Álvarez Sivana y Lucio Layme Villegas; los tamboreros, Martín Molina Valencia y Gumercindo Ccorahua Achinquipa; el bombero, Gregorio Sivana Solís y las waqras, Hipólito Ccorpuna Salazar, Teodoro Ccorpuna Salazar y Nicanor Lima Patiño.

\section{Reconocimientos}

Este texto fue realizado participativamente con la valiosa colaboración de los músicos tradicionales Cecilio Jaquis, Celestino Alarcón, Eusebio Villena, José Villena, Simeón Alarcón y Tiburcio «Lucho» Triveño; las bandas típicas Los Auténticos de Marka Marka, Huamanripaq, Santo Tomás, Nietos de Taukamarka, Acuarios de Callarani y Llaqtay Fiesta, banda que cordialmente me permitió la grabación y difusión de todas las muestras musicales que acompañan este trabajo. Asimismo, agradezco a los carguyuq Esperanza Ibarra, Mario Castro, Delfina Zamata, Wilfredo Abarca, Presentación Saldivar, Julio Retamozo, René Retamozo, Viviana Layme, Óscar Sivincha, Olimpia Huancahuiri y Telésforo Abrigo por permitirme ser parte de sus cargos; a Inés Adelaida Quispe e Hilario Arqque por su apoyo en la revisión de los textos en idioma quechua; a Jimmy Vera y Jorge Alejandro Vargas por sus precisiones en los textos en idioma castellano, y a la Dirección Desconcentrada de Cultura de Cusco, Ministerio de Cultura del Perú, por el respaldo brindado durante esta investigación. 


\section{REFERENCIAS BIBLIOGRÁFICAS}

Bolaños, César (1985). La música en el antiguo Perú. En La música en el Perú (pp. 1-64). Lima: Patronato Popular Pro Música Clásica.

Castro, Jesús (1999). La ganadería en el Perú: ayer y hoy. Boletín. Museo de Arqueología y Antropología, 2(7), 12-14. Lima: Museo de Arqueología y Antropología.

Díaz, Rafael (2013). La flauta traversa del Nuevo Mundo surgió en Tiwanaku. Revista Musical Chilena, LXVII(219), 12-41. Santiago de Chile: Universidad de Chile. https://doi.org/10.4067/s0716-27902013000100002

Gobierno Regional Cusco (2013). Memoria descriptiva del informe comunal: Provincia Chumbivilcas. Cusco, Área Antropología.

Instituto Nacional de Cultura (1978). Mapa de los instrumentos de uso popular en el Perú. Lima: INC, Oficina de Música y Danza.

Latham, Alison (2008). Diccionario enciclopédico de la música. México, D.F.: Fondo de Cultura Económica.

Matos, José (1984). Desborde popular y crisis del Estado. El nuevo rostro del Perú en la década de 1980. Lima: Instituto de Estudios Peruanos.

Montes, Edmundo (2013). Etnohistoria y misceláneas de Llusco. Tomo I. Cusco: ValleGrafp.

Montes, Edmundo (2016). Las wankas de Chumbivilcas. Literatura quechua del Ande. Arequipa: Arte Link E.I.L.R.

Museo Nacional de Antropología y Arqueología (1981). Exposición «Música y danza en el Antiguo Perú». Lima: Museo Nacional de Antropología y Arqueología.

Pinto, Arturo (1993). El waqra phuku. Boletín de Lima, mayo, 87, 27-31. Lima.

Ráez, Manuel (1998). Los ciclos ceremoniales y la percepción del tiempo festivo en el valle del Colca (Arequipa). En Música, danzas y máscaras en los Andes (pp. 253-297). Lima: Instituto Riva-Agüero de la Pontificia Universidad Católica del Perú.

Ráez, Manuel (2002). En los dominios del cóndor: fiesta y música tradicional del valle del Colca. Lima: Instituto Riva-Agüero de la Pontificia Universidad Católica del Perú.

Robles, Román (2000). La banda de músicos. Las bellas artes musicales en el sur de Ancash. Lima: Universidad Nacional Mayor de San Marcos, Facultad de Ciencias Sociales, Instituto de Investigaciones Histórico Sociales. 
Roel, Josafat (1975). Entrevista, Lima, enero de 1975. En Fernando García (1978). Algunos aspectos de la trompeta en el Ande peruano (II), Boletín Música, 69, 3-19. marzo-abril. La Habana: Casa de las Américas.

Romero, Raúl (1985). La música tradicional y popular. En La música en el Perú (pp. 215-283). Lima: Popular Pro Música Clásica.

Romero, Raúl (1998). Cambio musical y resistencia cultural en los Andes centrales del Perú. En Música, danzas y máscaras en los Andes (pp. 21-60). Lima: Instituto Riva-Agüero de la Pontificia Universidad Católica del Perú.

Romero, Raúl (2008). Instrumentos musicales andinos. En Fiesta en los Andes: ritos, música y danzas del Perú (pp. 166-197). Lima: Fondo Editorial de la Pontificia Universidad Católica del Perú.

Tamayo, José (1978). Historia social del Cuzco republicano. Lima: Industrial.

Villena, Arturo (1987). Qorilazo y región de refugio en el contexto andino. Cusco: Papelería Peñarol. 